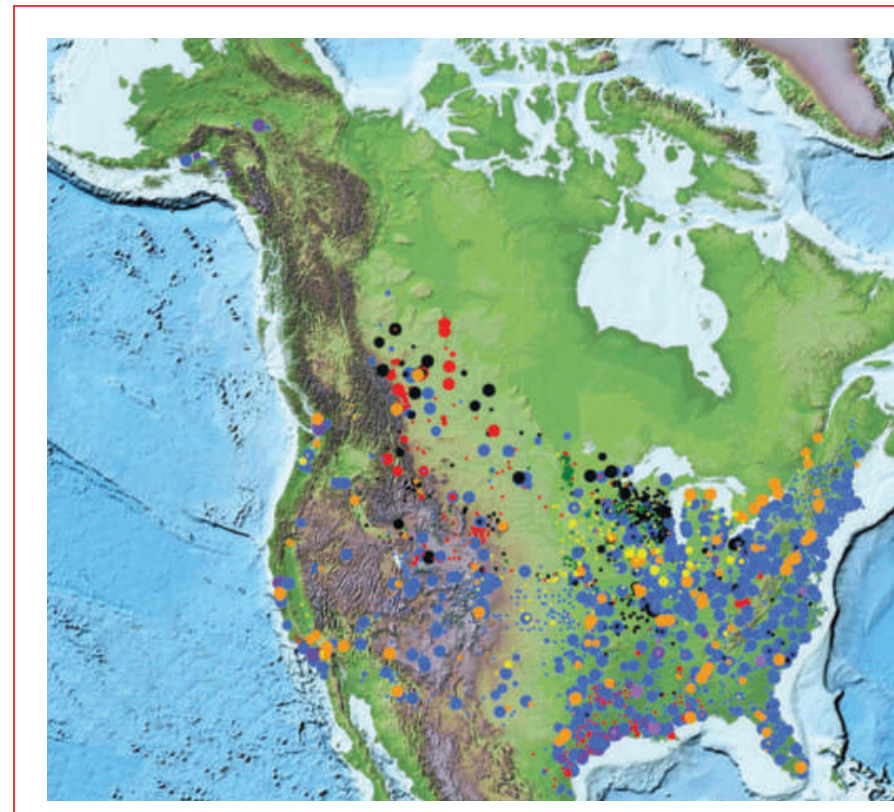

\section{SNAPSHOT Carbon stores}

A newly updated atlas from the US Department of Energy shows how North America might store its excess carbon dioxide - if it could figure out how to do so.

On the left is a map showing many of the known stationary
$\mathrm{CO}_{2}$ sources in the United States and Canada. Blue represents electricity generation, with many power plants clustered in the east and midwest. Orange represents cement manufacturing, including groupings in southern California. Red indicates petroleum and natural-gas processing, showing up most starkly as the Alberta tar sands in Canada.

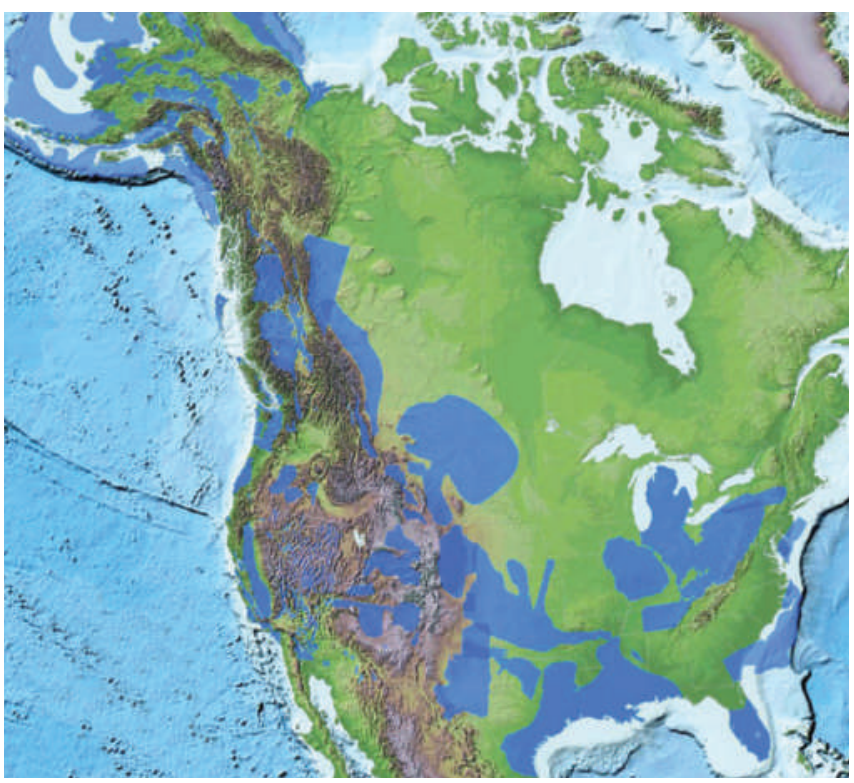

Potential reservoirs for sequestering much of the $\mathrm{CO}_{2}$ from such activities could be deep saline formations (above right, in blue), or layers of rock permeated with brine. The new atlas estimates that saline formations could hold anywhere from 920 billion-3,400 billion tonnes of $\mathrm{CO}_{2}$. This dwarfs the potential for existing oil and gas reservoirs ( 82 billion tonnes) or unmineable coal seams (156 billion-184 billion tonnes).

Yet turning saline formations from dream reservoir into sequestration reality remains a challenge. In Alberta, a consortium is in the process of identifying potential formations for a test project to begin in 2009. Alexandra Witze

\title{
Electron 'bump' may confirm dark matter
}

A high-altitude balloon experiment above the Antarctic seems to have seen a possible signature of mysterious 'dark matter', similar to that spotted earlier this year by a European satellite.

The Advanced Thin Ionization Calorimeter (ATIC), an experiment to search for charged particles from space, has spotted a surplus of high-energy electrons coming from somewhere in the cosmos (see Letter, page 362, and News \& Views, page 329). Although the interpretation is far from certain, the electrons could be produced by dark matter - previously undetected particles that physicists believe make up $85 \%$ of all matter in the Universe.

ATIC's findings are similar to data from the PAMELA (Payload for Antimatter Matter Exploration and Light-nuclei Astrophysics) satellite mission, a collaboration between Italy, Russia, Germany and Sweden that spotted an excess of high-energy positrons, or anti-electrons, at roughly similar energies (see Nature 454, 808; 2008). "In several respects the two measurements complement each other," says John Wefel, head of the ATIC collaboration and a physicist at Louisiana State University in Baton Rouge.

Wefel's team looked at data from two multiday ATIC missions flown between 2000 and 2003 some 35 kilometres above the Antarctic

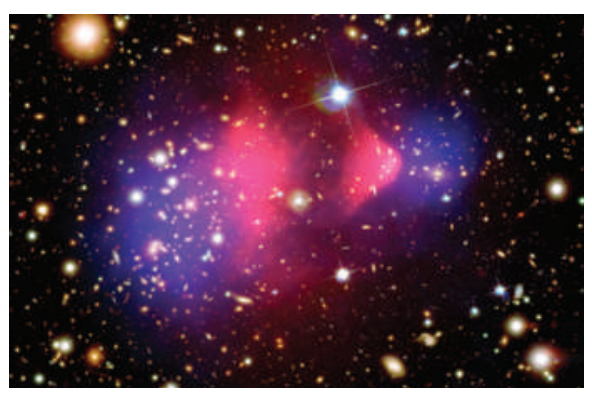

Several experiments may have found dark matter, the existence of which is inferred here (blue). ice. With $99.5 \%$ of Earth's atmosphere beneath them, the missions measured electrons that come from various galactic sources such as exploding stars. As predicted by theory, the experiment saw fewer electrons at higher energies. But between 300 gigaelectronvolts and 600 gigaelectronvolts, the number of electrons rose sharply before falling off to background levels.

PAMELA's results, as posted on the arXiv $\frac{\frac{\pi}{2}}{2}$ preprint server last month (O. Adriani et al. http://arxiv.org/abs/0810.4995; 2008) and also submitted to Nature, show positrons increasing up to 100 gigaelectronvolts. Although the collaboration did not report beyond this energy level, some suspect that the number of positrons may continue to increase at higher energies. And, because the PAMELA and ATIC data were measured in different ways, researchers believe that their findings probably confirm each other. 
The 'bump' ATIC sees in the number of electrons could be the result of heavy darkmatter particles annihilating, according to Wefel. When this happens, two dark-matter particles collide and their mass is converted to pairs of fast-moving electrons and positrons, the energies of which would correspond to the mass of the original particles.

"That's certainly the sexiest of the possibilities," says Dan Hooper, a theoretical physicist at Fermi National Accelerator Laboratory in Batavia, Illinois. The exact nature of the dark-matter particles that produce electrons is uncertain, but one idea is that they may be ordinary particles that spend part of their lives in a compact extra dimension of space. Whereas the particles would appear relatively stationary to observers trapped in three spatial dimensions, they could be moving at ultra-high speeds in a fourth spatial dimension. At high speeds, they would create a gravitational force that could be felt by matter trapped in three dimensions of space-time. "It's very wild," Hooper says.

Other, more mundane reasons might also "Annihilation explain the data. The leading candidate of dark-matter is a nearby pulsar, particles is says Aldo Morselli, certainly the a particle physicist sexiest of the from the Italian possibilities." of Nuclear Physics at the University of

Rome, Tor Vergata. Pulsars, the fast-spinning remnants of supernovae explosions, have enormous magnetic fields that can accelerate electrons to the high energies seen in the experiments, although ATIC's bump is harder to match to a pulsar than PAMELA's increase.

Wefel agrees that it is too early to say for certain whether ATIC and PAMELA have seen dark matter. "You cannot make a hard and fast claim," he says. "The case is still open."

But more evidence may come soon. NASA's Fermi telescope (formerly known as GLAST), which launched in June this year, is designed to hunt for high-energy $\gamma$-rays. But the telescope can also spot electrons and positrons, according to Morselli. In the coming months, he predicts that the telescope will be able to verify the positron and electron data of PAMELA and ATIC. In addition, Fermi may be able to spot $\gamma$-rays that have come from dark-matter annihilations. "By the spring of 2009, we'll have a lot more information," Morselli says. Geoff Brumfiel

늠

METHANE ON MARS

Engineers rule out gassy landing spot as too risky. www.nature.com/news

\section{Middle East synchrotron on the lookout for funds}

The Middle East's first synchrotron facility, a collaborative effort intended to promote peace through international scientific cooperation, formally opened its doors on 3 November in Allan, Jordan. But unless the project can plug a $115-$ million (US\$19-million) shortfall, the machine's completion risks being stalled.

Following a flurry of behind-the-scenes discussions, officials are confident that they will find the money. The facility's inauguration at Al-Balqa Applied University may have helped: when ministers and other representatives finally saw the buildings, and the beamlines and other machinery being installed, it had "a big psychological impact," says Herwig Schopper, who retired as head of the project council on 4 November.

The idea of a synchrotron as a bridge for peace in the Middle East was first proposed in 1997; two years later, SESAME (Synchrotron-light for Experimental Science and Applications in the Middle East) launched as an autonomous international research organization, under the auspices of the United Nations Educational, Scientific and Cultural Organization (UNESCO). It is modelled on CERN, Europe's particle-accelerator centre near Geneva, Switzerland. Jordan won the bid to host it.

Most of SESAME's technical equipment comes from donations from facilities being replaced or upgraded elsewhere. Its 0.8 gigaelectronvolt (GeV) injector system comes from BESSY I in Berlin, Germany, and entire beamlines from the Daresbury Synchrotron Radiation Source in the UK, the Laboratoire pour I'Utilisation du Rayonnement Électromagnétique (LURE) in Orsay, France, and the Swiss Light Source in Villigen. In addition, powerful magnets and other equipment are being loaned by the Lawrence Berkeley National Laboratory and the SLAC National Accelerator Laboratory, both in California. SESAME is supposed to be completed by 2011 , with a price tag of roughly $\$ 100$ million, which includes labour and the value of donated parts, says Chris Llewellyn-Smith, the new head of the SESAME council.

But instead of the $1 \mathrm{GeV}$ machine initially proposed for the site, SESAME members decided several years ago to opt for an ambitious $2.5 \mathrm{GeV}$ machine, comparable to new third-generation sources elsewhere. The project members Bahrain, Cyprus, Egypt, Israel, Iran, Jordan, Pakistan, the Palestinian Authority and Turkey - agreed to pay for the increase in operational costs, which will rise progressively from $\$ 1.3$ million to $\$ 5$ million annually over the next four years. The problem is funding the capital costs of the $2.5 \mathrm{GeV}$ storage ring.

Several possible solutions seem to be emerging. Jordan is said to be offering a loan to prevent delays. As the host country, it has 